\title{
Area Model and Dimensioning Guidelines of Multi-source Energy Harvesting for Nano-micro Interface
}

\author{
Raul G. Cid-Fuentes, Student Member, IEEE, Albert Cabellos-Aparicio, Member, IEEE \\ and Eduard Alarcón, Member, IEEE
}

\begin{abstract}
Multi-source energy harvesters is a promising, robust alternative to power the future Internet of nano things (IoNT), since the network elements can maintain their operation regardless of the fact that one of its energy sources might be temporarily unavailable. Interestingly, and less explored, when the energy availability of the energy sources present large temporal variations, combining multiple energy sources reduce the overall sparsity. As a result, the performance of a multiple energy harvester powered device is significantly better compared to a single energy source even if they harvest the same amount of energy. In this context, a framework to model and characterize the area for multiple source energy harvesting powered systems is proposed. This framework takes advantage of this improvement in performance to provide the optimal amount of energy harvesters, the requirements of each energy harvester and the required energy buffer capacity, such that the overall area or volume is minimized. On top of these results, self-tunable energy harvesters are explored as a solution and compared to multi-source energy harvesting platforms. As the results show, by conducting a joint design of the energy harvesters and the energy buffer, the overall area or volume of an energy harvesting powered device can be significantly reduced.
\end{abstract}

\section{INTRODUCTION}

Nanotechnology is providing a new set of tools to the engineering community to integrate communicating nanosensors. By means of communication, these nanosensors will be able to achieve complex tasks in a distributed manner [2]. The resulting nanonetworks will enable unique applications. For the time being, the communication options for nanosystems are very limited due to large constraints that these nanosensors face with regard to energy availability.

Recent advancements in electronics [2], [3] have pointed out that energy harvesting $(\mathrm{EH})$ is a firm candidate as the key enabling technology in the development of nanonetworks with perpetual character. These upcoming networks, show unique properties not only because of ultra-low power constraints but also because of the fact that the energy state is time varying. This is, the energy buffer (e.g. a supercapacitor or a battery) is constantly charging and discharging in a random manner [4]. For this reason, one of the main challenges in the design of such devices lies in the dimensioning of both the energy harvesting and energy buffer units [4]. Considering both subsystem units to be sufficiently large solves undesired

The authors are with the N3Cat (NaNoNetworking Center in Catalunya), Universitat Politècnica de Catalunya (UPC), 08034 Barcelona, Catalunya, Spain (e-mail:rgomez@ac.upc.edu, acabello@ac.upc.edu, eduard.alarcon@upc.edu).

A preliminary version of this work was presented in [1].

Copyright (c) 2012 IEEE. Personal use of this material is permitted. However, permission to use this material for any other purposes must be obtained from the IEEE by sending a request to pubs-permissions@ieee.org.

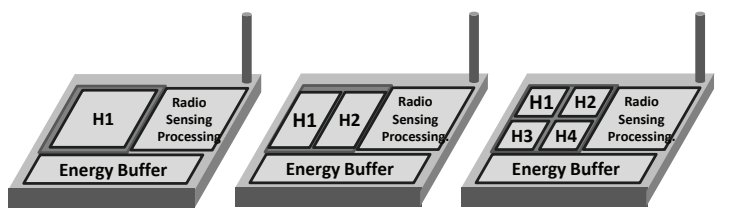

Fig. 1. Multi-source Energy harvesting enabled nano-micro interface. Increasing the number of sources reduces the efficient area for harvesting but maximizes the probability of finding an active energy source.

interruptions during the normal operation of the nanosensor and, accordingly, on the nanonetwork. However this comes at the cost of precluding desirable miniaturization of the nanosensors, caused by the relatively small power densities of existing ambient energy sources and low energy density of energy buffers [5], [6]. As an example, in order to harvest $0.2 \mathrm{~mW}$ vibrational energy and to store $1 \mathrm{~J}$ of energy, an energy harvester of approximated $1 \mathrm{~cm}^{2}$ and an energy buffer of approximated $2 \mathrm{~cm}^{3}$ would be required.

Recently, multi-source energy harvesters are gaining interest as a robust alternative to power wireless sensors [7]. To implement multi-source energy harvesters, there appear two feasible approaches. On the one hand, these can be implemented through platforms which combine a few number of energy harvesters, each devoted to each source of energy [7], [8], [9]. On the other hand, self-tunable approaches permit tuning their oscillating frequency, therefore enabling multiband capabilities to harvest energy from multiple energy sources [10], [11].

These platforms are more robust than the single-source ones. Indeed, if a certain energy source renders unavailable for a certain time period, due to the time asynchronicity among energy sources the sensor node can still maintain its normal operation. An additional, but less explored, advantage of heterogeneous multiple-source energy harvesters, which aids the miniaturization of the sensor nodes, is that when the ambient energy presents large temporal variations (i.e., the harvested power randomly varies over a wide range during time) the combination of multiple statistically independent energy sources lowers the sparsity of the overall energy which is harvested. This causes that devices, which are powered by multi-source energy harvesters show lower outage probabilities in contrast to single-source configurations. Equivalently, the requirements in terms of energy buffer capacity can be relaxed while maintaining the same performance. As an example, Fig. 1 shows three wireless motes that implement one, two or four energy harvesters which occupy the same overall area, in a chip-like planar implementation. 
In this paper, we present an analytical framework to model the overall occupied area by the energy harvesting and energy buffer units. In particular, this model accounts for the requirements and capabilities of the wireless mote, and is useful to provide (i) the optimal number of energy harvesters, (ii) their size and (iii) the energy buffer capacity, such that the overall area of the wireless communicating device is minimized, while still meeting the user-defined requirements of the communications unit. On top of these results, we explore the capabilities of self-tunable energy harvesters as a feasible alternative to multi-source platforms [10]. In this context, we evaluate their performance in terms of harvested power and compare it to the performance of multi-source energy harvesting platforms.

To evaluate the provided model, we focalize on the design of the nano-micro interface [12]. This network element stems as the interface between the nanonetwork and the macroscale network. As such, nano-micro interfaces show larger requirements in terms of computation and communications capabilities and, therefore, these systems present larger power consumption as well as overall size. Notice, however, that this model can be scaled down to the size of a nanosensor, by assuming the detailed constraints of such devices.

This framework shows that harvesting energy from multiple sources by using either multi-source platforms or selftunable energy harvesters provides significant improvements in energetically sparse scenarios. These improvements, jointly considered with an optimal dimensioning of the energy buffer will pave the way to smaller energy management units and, therefore, actual miniaturization of eventual nanonetworking devices. The paper is structured as follows: In Sec. II-A we present the sparse energy sources. In Sec. III we compare the performance of single-source to multi-source energy harvester powered devices. In Sec. IV we present the circuit area model to be optimized while in Sec. V we evaluate this model in a particular case. In Sec. VI we explore the capabilities of selftunable energy harvesters. Finally, in Sec. VII we conclude our work.

\section{OVERVIEW}

In this section we overview the properties of the environmental energy and define the metrics to evaluate the results of this work.

\section{A. Sparse Energy Sources}

Ambient energy is generally generated by the aggregation of an extensive number of physical entities which simultaneously radiate power [5]. Then, the random contribution of each entity, in both magnitude and time duration, entails a timevarying character in the aggregated power.

Accordingly, we refer to any physical phenomena which produces an aggregated power in a sparse, time-varying manner, such that this power cannot be known or estimated and the magnitude of the instantaneous power falls within a wide range, as a sparse energy source. In fact, sparse energy sources are present in a wide variety of physical phenomena. Among others, acoustic energy, mechanical, vibrational or RF

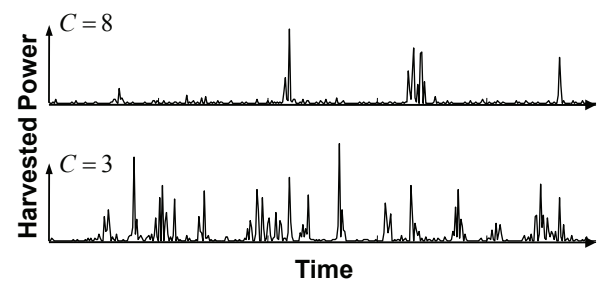

Fig. 2. Harvested power from a sparse ambient source of peak power to average power ratio of (upper) $C=8$ and (lower) $C=3$.

energy [13], [14], [15] are considered representative examples of such sources, when considering a large time scale.

In this work, we propose the peak power to average power ratio as a metric to enable the comparison of performance of ambient energy sources. This metric is given by:

$$
C=\frac{P_{p e a k}}{P_{H}},
$$

where $P_{\text {peak }}$ is the average peak power and $P_{H}$ refers to the average harvested power. Fig. 2 shows examples of two random energy sources with different peak power to average power ratio $(C=8$ and $C=3)$. As it is shown, energy sources with large peak power to average power ratios are characterized by short but powerful bursts of energy, while leaving large inter-burst times where the available energy is far below the average value. On the contrary, energy sources with low values of this metric are characterized by being more constant and predictable.

\section{B. Evaluation Metrics}

We use the energy utilization as a main metric to relate the occupied area of an energy harvester, its harvestable power and the required performance of the nano-micro interface. The energy utilization provides a link between the energy model, the environmental harvested power, the network requirements and the energy buffer capacity. This is defined as:

$$
\rho_{e}=\frac{P_{C}}{P_{H}} .
$$

where $P_{H}$ is the harvested power and $P_{C}$ stands for the required power to perform a certain application. The energy utilization is evaluated in the Energy-Erlang units [4].

Secondly, we use the energy outage probability, $p_{\text {out }}$, as a metric to evaluate the performance of the nano-micro interface. The energy outage is defined as the time interval during which the device node does not have enough stored energy, and thus its operation is temporarily interrupted.

\section{Multiple Source Energy Harvesters}

Multi-source energy harvesters are able to combine the energy from multiple energy sources. This reduces the chances that the nano-micro interface is in a deep energy fading, where it is not able to harvest energy for a significant amount of time, since whenever an energy source is faded, any other energy source can be supplying energy. In other words, combining independent energy sources, the sparsity of the overall process is reduced and thus the energy fadings are potentially reduced, 


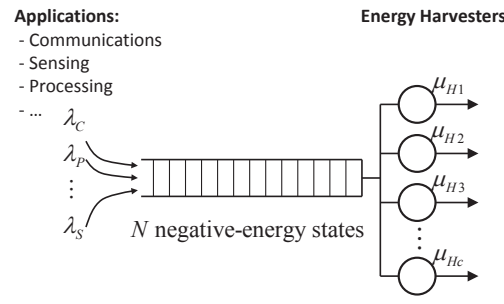

Fig. 3. The negative energy queue model.

as well. In this section we provide a model for multi-source energy harvester platforms and we evaluate the improvement on performance that using multiple energy harvesting platforms has when contrasted to single harvester platforms.

\section{A. Energy Model}

In order to evaluate the performance, we use the negative energy queue model [4], which is shown in Fig. 3. This Markov-based model is similar to other existing energy models for energy harvesting [16], [17], [18], [19]. However, as it is shown, this model pursues to model an energy harvesting powered nano-micro interface as a classical communications queue. That is, $(i)$ the stability condition must be $\rho_{e}<1$, (ii) the idle state is defined as the state of having an empty queue and (iii) the loss of communication is assigned to a full queue.

This model considers that the arrivals of this queue are generated by the set of applications of the nano-micro interface node. i.e., every time an application spends one unit of energy, it generates an arrival of negative energy. Each kind of application has an associated generation rate in power units (e.g. $\lambda_{C}$ for communications, $\lambda_{P}$ for processing and $\lambda_{S}$ for sensing). On the other hand, each harvester has an associated service time, $T_{H}=E_{s} / \mu_{H}$, which is the time that this energy harvesting unit needs to process one negative energy packet, where $E_{s}$ is the energy of a negative energy packet and $\mu_{H}$ the energy harvesting rate in power units. We find that this time is characterized by a random variable defined as:

$$
t_{H} \equiv \text { time s.t. } \int_{t_{H}} P_{H}(t) d t=E_{s} .
$$

Thus, the number $N$ of negative energy states is related to the energy buffer capacity, $C_{B}$ as:

$$
N=\frac{C_{B}}{E_{s}} .
$$

Additionally, if at a certain time $t_{k}$ the queue has $L_{k}$ negative energy packets, then the energy state $s_{k}$ at the energy buffer is given by:

$$
s_{k}=C_{B}-L_{k} E_{s} .
$$

This models brings significant benefits to model multisource energy harvesters. In particular, the negative- energy queue model is able to easily handle multiple energy harvesters, by connecting them in parallel, such as a communication queue with multiple servers (e.g. M/M/c/N, M/G/c/N and $\mathrm{G} / \mathrm{G} / \mathrm{c} / \mathrm{N})$.
In order to exemplify this, if we assume a single-source energy harvester the outage probability can be easily calculated by means of queue theory on $\mathrm{M} / \mathrm{G} / 1 / \mathrm{N}$ :

$$
p_{\text {out }}=P_{N}=1-\frac{1}{\pi_{0}+\rho_{E}}
$$

where $\pi_{0}$ refers to the probability that there are 0 negativeenergy packets left within the queue right after the last negative-energy packet was processed by the energy harvester. As such, it is only required to estimate the probability of having a depleted queue. In particular, $\pi_{0}$ is found as a solution for:

$$
\begin{gathered}
\pi_{n}=\sum_{n=0}^{N-1} \pi_{j} p_{j n}, \quad 0 \leq n \leq N-1 \\
\quad \text { and } \quad \sum_{n=0}^{N-1} \pi_{n}=1
\end{gathered}
$$

where, equivalent to $\pi_{0}, \pi_{n}$ refers to the probability that there are $n$ negative-energy packets left and $p_{j n}$ stands for the state transition probability of remaining negative-energy packets from the state $j$ to the state $n$, considering each state right after a negative-energy packet has been processed by the energy harvester.

\section{B. Performance of a Multiple Source Energy Harvester}

We focus on the nano-micro interface to evaluate the provided model. A nano-micro interface is expectedly larger than the remaining nanosensors, since these must operate as a network interface between the nanonetwork and the macroscale environment. for these devices, we have considered an average communications rate of $\lambda_{c}=P_{C}=100 \mu \mathrm{W}$. Then, we have considered each negative energy packet to be of $10 \mu \mathrm{J}$. Finally, we have set the overall harvesting rate $N \mu_{H}=P_{H}=P_{C} / \rho$, where $\rho_{e}$ has been set as an evaluation parameter. Therefore, each harvester harvests an average power of $P_{C} / \rho_{e} N$. These energy harvesting rates can be achieved by means of vibrational harvesters [5].

In order to generate the sparse energy sources, we have approximated the ambient energy by a random process generated by exponentially distributed energy bursts of power $P_{H} C / N$, with an inter-burst time of $0.1 / C$ seconds. An exponentially distributed random process has been chosen as it presents the largest entropy, thus estimating the worst case [13].

Fig. 4 and Fig. 5 compare the improvement over $p_{\text {out }}$ that using multiple harvesters has as a function of the energy buffer capacity, $C_{B}$, for a peak power to average power ratio of $C=$ 10 and $C=100$ respectively. These results have been obtained by assuming in the negative energy queue model $\rho_{e}=0.9$. As it is shown, there is a clear improvement, since varying from one to five harvesters, the energy buffer capacity can be reduced from $30 \mathrm{~mJ}$ to just $5 \mathrm{~mJ}$ and from $600 \mathrm{~mJ}$ to just $100 \mathrm{~mJ}$, while still maintaining $p_{\text {out }}<10^{-3}$.

In addition to this, Fig. 6 and Fig. 7 compare this improvement as a function of the $\rho_{e}$ for peak power to average power ratios of $C=10$ and $C=100$ respectively. In order to obtain these results, the energy buffer capacity has been set to $C_{B}=10 \mathrm{~mJ}$ in Fig. 6 and to $C_{B}=100 \mathrm{~mJ}$ in Fig. 7. As it 


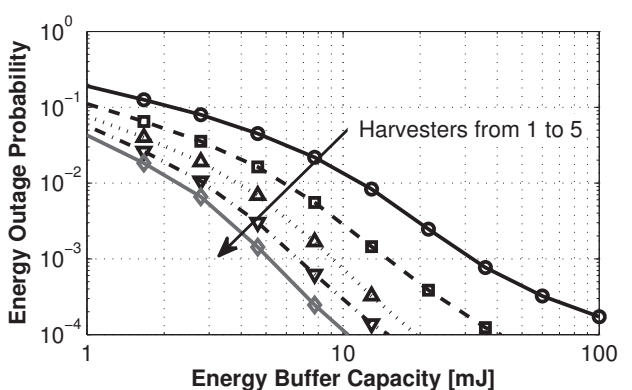

Fig. 4. Energy Outage Probability as a function of the energy buffer capacity. $\rho_{E}=0.9 \mathrm{E} 2$ and $C=10$.

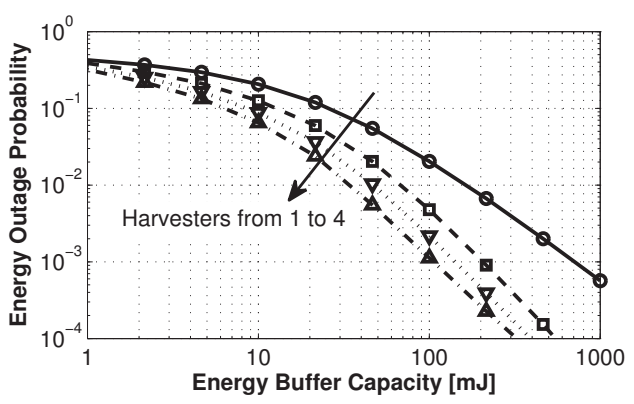

Fig. 5. Energy Outage Probability as a function of the energy buffer capacity. $\rho_{E}=0.9 \mathrm{E} 2$ and $C=100$.

is shown, multi-source energy harvesters are able to provide similar performance, but at larger $\rho_{e}$ values and, therefore, requiring smaller energy harvesting area.

As a result, we observe that multi-source energy harvesters can help reducing both the energy buffer capacity, as well as the energy harvesting requirements, while still providing the required performance.

\section{Circuit Area Model}

As seen in the previous section, additional energy harvesters have a positive impact upon the performance. Nonetheless, this technique produces a non-negligible area overhead, since each energy harvester requires some additional circuitry and separation space.

An additional compromise is that low values of $\rho_{e}$ help reducing the energy buffering capacity at the cost of proportionally increasing the energy harvesting requirements.

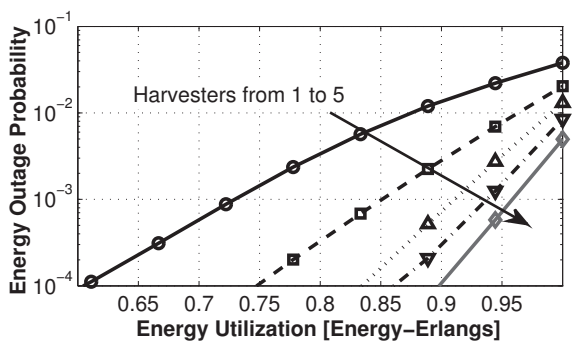

Fig. 6. Energy Outage Probability as a function of the energy utilization. $C_{B}=10 \mathrm{~mJ}$ and $C=10$.

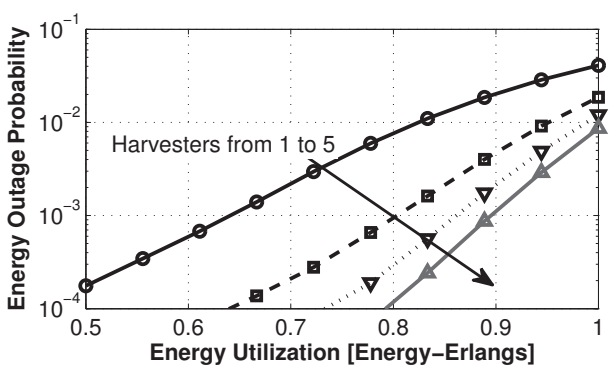

Fig. 7. Energy Outage Probability as a function of the energy utilization. $C_{B}=100 \mathrm{~mJ}$ and $C=100$.

These compromises motivate a framework for circuit area optimization which considers the user-defined requirements, the area overhead of multiple harvesters and the energy buffer capacity. In order to do so, we first relate the required power, harvesting power, number of harvesters and energy buffer capacity which are able to achieve the required performance in energy outage probability, through the energy model presented in Sec. III. Afterwards, this is translated into circuit area by means of the following model.

We then define the overall area of the system as:

$$
A_{\text {TOTAL }}=A_{H}+A_{B}+A_{A},
$$

where $A_{H}$ refers to the area of the harvesting unit, $A_{B}$ stands for the area of the energy buffer unit and $A_{A}$ is the area of the applications units (i.e., processing, sensing and communications unit). In particular, since $A_{A}$ is fixed and provided by a certain application, $A_{A}$ is not considered in the following circuit area optimization.

\section{A. Area of the Energy Harvesting Unit}

The area of the harvesting unit depends on mainly two factors, the number of energy harvesters and the power that these aim to harvest. As shown in [5], the ambient power is generally characterized by a given power density. As such, the overall area is expectedly proportional to the desired power to be harvested. Alternatively, integrating more than one energy harvester requires additional circuitry, which increases the eventual size of the unit. In this work, we linearly approximate the area of the energy harvesting unit in terms of the number of energy harvesters and desired power rate:

$$
A_{H}=A_{H 0}+A_{H N} N_{H}+A_{H P} P_{R} / \rho_{e},
$$

where $A_{H 0}$ refers to a constant area, $A_{H N}$ to the partial contribution of $A_{H}$ with respect to the number, $N_{H}$, of energy harvesters and $A_{H P}$ to the partial contribution of $A_{H}$ with respect to the required power $P_{H}$.

The considered values in this works are shown in Table I. These correspond to reasonable values that have previously been reported [5].

\section{B. Area of the Energy Buffer}

In line with recent advancements in energy buffering [6], each technology presents an associated energy density. In this context, we have considered consistent values for this density 
TABLE I

VALUES USED IN THE OPTIMIZATION FRAMEWORK

\begin{tabular}{l|c|c}
\hline Parameter & Value & units \\
\hline$A_{H 0}$ & 0.01 & $\mathrm{~cm}^{2}$ \\
$A_{N H}$ & .01 & $\mathrm{~cm}^{2}$ \\
$A_{N P}$ & 6.66 & $\mathrm{~cm}^{2} \mathrm{~mW}^{-1}$ \\
\hline
\end{tabular}

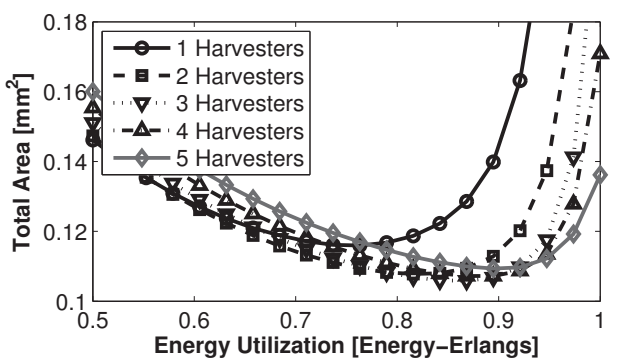

Fig. 8. Overall Area in terms of the Energy Utilization. $C=10$.

of $D_{B}=2 \mathrm{~J} / \mathrm{cm}^{3}$ and a fixed height of $1 \mathrm{~cm}$. Similar to $A_{H}$, we may linearly approximate the overall area of the energy buffer as:

$$
A_{B}=A_{B 0}+C_{B} D_{B},
$$

where $A_{B 0}$ is a fixed area overhead and $C_{B}$ is the required capacity of the energy buffer in $\mathrm{mJ}$ units.

\section{Evaluation of the Area Model}

In order to optimize the area, we have simulated the nanomicro interface through the same energy model as described in the previous sections. Then, we have assumed a tolerable performance of a wireless device, when its energy outage probability is below $p_{\text {out }}=10^{-4}$.

We show in Fig. 8 the overall occupied area for the joint energy harvesting and energy buffer unit, such that the user defined requirements in terms of output power and energy outage probability are met. This area corresponds assuming that the environmental energy is characterized by a peak power to average power ratio of $C=10$. As it is shown, the overall area is shows an optimal minimum for $\rho_{e}=0.87 \mathrm{E} 2$. This is due to the fact that for fixed values of power requirements, a large energy utilization ratio reduce the amount of harvested energy, therefore reducing the size of the energy harvester. However, this reduction of the energy harvester comes at the price of increasing the size of the energy buffer.

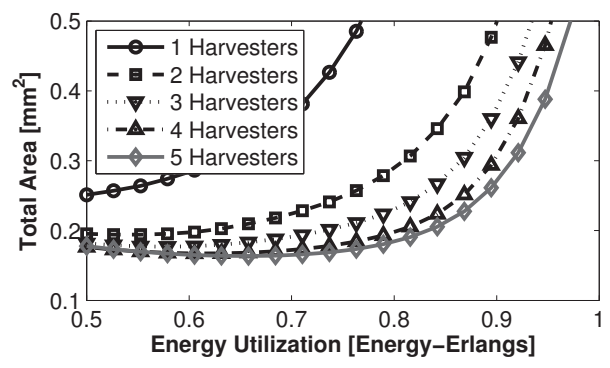

Fig. 9. Overall Area in terms of the Energy Utilization. $C=100$.
TABLE II

COMPONENT REQUIREMENTS

\begin{tabular}{c|c|c|c}
\hline$C$ & Parameter & Value & Units \\
\hline \hline 10 & Harvesters & 4 & - \\
& Area Harvester (total) & 7.7 & $\mathrm{~mm}^{2}$ \\
& $P_{H}$ (each) & 27.7 & $\mu \mathrm{W}$ \\
& Area Energy Buffer & 3 & $\mathrm{~mm}^{2}$ \\
& Capacity Energy Buffer & 15 & $\mathrm{~mJ}$ \\
\hline 100 & Harvesters & 5 & - \\
& Area Harvester (total) & 8.3 & $\mathrm{~mm}^{2}$ \\
& $P_{H}$ (each) & 40 & $\mu \mathrm{W}$ \\
& Area Energy Buffer & 5 & $\mathrm{~mm}^{2}$ \\
& Capacity Energy Buffer & 25 & $\mathrm{~mJ}$ \\
\hline
\end{tabular}

Similarly, Fig. 9 shows the results of the circuit area optimization when considering the same system requirements, but assuming a peak power to average power of $C=100$. As it is shown, an increase in this ratio enlarges the size of the overall area, regardless of the number of energy harvesters and their operation point. This increase is caused by the fact that the nano-micro interface runs on the stored energy for a longer time. In this case, it is found that increasing the number of energy harvesters shows a significant benefit, since the sparsity of the energy is reduced. In particular, the minimum area is found at a $\rho_{E}=0.66 \mathrm{E} 2$, considering five energy harvesters. The outcomes of this design, which are required for the energy harvesting unit and an energy buffer to minimize the area can be found in Table. II for both cases.

\section{Self-tunable Multi-band Energy Harvesters}

In case that the considered energy sources are of the same type and the differences among them is that each is produced at a different frequency band, self-tunable energy harvesters emerge as an encouraging alternative to multisource platforms. These devices have the property of tuning their oscillating frequency over a wide range to adapt it to the frequency band of the harvestable energy [10].

This technology aims to provide a much higher performance compared to independent multi-source platforms in cases where the ambient energy is very sparse and the frequency bands are uncorrelated to each other. In this case, a single energy harvester can generate more power than small energy harvesters. However, this improvement compared to multisource platforms is not always achieved because of two main reasons. On the one hand, when the different bands generate power simultaneously, self-tunable energy harvesters can only tune a one of the frequencies, thus disregarding the other bands. On the other hand, a similar concept to cognitive-radio communications [20], these devices must implement spectrum sensing techniques to detect which frequency band generates a larger amount of power, therefore requiring power to generate power.

To exemplify this, consider the time diagram shown in Fig. 10. In the figure, two IoNT platforms (one equipped with a multi-source platform, and one equipped with a selftunable harvester) harvest power from bands \#1 and \#2. We consider that both platforms integrate an energy harvester of the same overall occupied area. Therefore, the self-tunable energy harvester integrates a single energy harvester which 


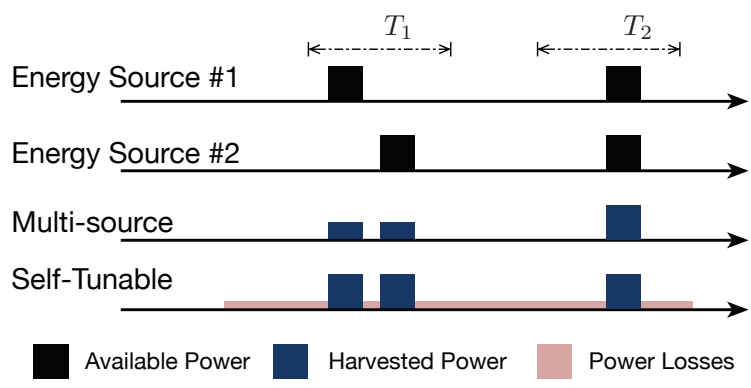

Fig. 10. Comparison between multi-source and self-tunable platforms.

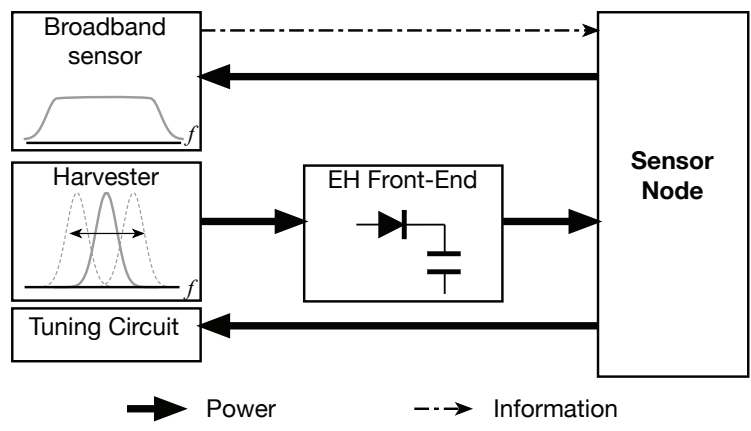

Fig. 11. Generic block diagram of an energy harvesting powered device that employs a self-tunable energy harvester.

can select the operating frequency band, whereas the multisource energy harvester is divided by two energy harvesters, one for each frequency band. Then, we observe that during the time $T_{1}$ both energy sources generate power at different times, whereas during $T_{2}$ the energy sources simultaneously generate power. As a result, the self-tunable energy harvester shows potential improvement during $T_{1}$ since it can harvest twice power, whereas the multi-source platform scavenges more energy during $T_{2}$ since both harvest the same amount of power while this does not requires to spend power in sensing the environment.

In this section we provide a generic model for a self-tunable energy harvester and provide compare their performance to multi-source approaches as a function of critical factors which affect their performance.

\section{A. A Self-tunable Energy Harvester}

We show a generic model block diagram of a self-tunable energy harvester in Fig. 11. This is composed of four sub-units, namely the broadband sensor, harvester, energy harvesting front-end and tuning circuit. As the figure shows, the harvester is the only sub-unit which generates power, whereas the remaining units require power to realize their operation. We define the net harvested power as the net contribution of power generated by the harvester, broadband sensor and tuning circuit:

$$
P_{H}=\eta P_{E H}(t, B)-P_{B}-P_{T}
$$

where $\eta$ stands for the efficiency of the EH front-end, $P_{E H}$ is the power generated in the harvester sub-unit, which is tuned at the band $B, P_{B}$ refers to the required power from the broadband sensor to operate, $P_{T}$ stands for the power which is consumed in the tuning circuit. As it follows we briefly describe the operation of each unit.

1) Harvester: The tunable energy harvester stems as the key element in the energy harvesting unit. This is the only component which generates energy by converting environmental energy into electric current. This component has tunable properties, i.e., its oscillating frequency can be modified by adjusting its electrical parameters. Provided that this component generates energy, there is a direct relation between its occupied area and the power that it is able to harvest. As such, it is desired that this component occupies the largest area allocated for the energy harvesting unit. The harvested power is given by:

$$
P_{E H}(t, B)=(S(t) * h(t, B)) A_{e f f},
$$

where $S$ is the spectral power density of the available energy source, in power/area units, $h(t, B)$ stands for the transfer function of the harvester, which is tuned to the band $B$, and $A_{\text {eff }}$ refers to the effective area of the harvester.

2) Broadband Sensor: In order to choose the optimal oscillating frequency of the energy harvester, a broadband sensor is integrated to detect most powerful band.These devices show remarkable properties to detect oscillations at a significantly wide frequency range. Unfortunately, they cannot be used as energy harvesters. As it is shown in Fig. 11, this unit requires a supply power to operate and to reports the sensed information. The nano-micro interface must integrate spectrum sensing tools to process this information to decide whether to re-tune the harvester. The power consumed by this unit, $P_{B}$ is assumed constant during the normal operation of the device.

3) Tuning Circuit: This circuit accommodates the natural frequency of the energy harvesting depending on the processed results retrieved by the sensed data of the broadband sensor. The basic element of this circuit is a capacitor. By selecting a capacitor voltage, $V_{C}$, the natural frequency of the energy harvester is tuned to a different frequency. Recent studies show approximately linear dependency between the frequency and this voltage [10]. As such, the tuned band $B$ is selected according to:

$$
B=k f_{0} V_{C},
$$

where $k$ is a given constant, $f_{0}$ is the center frequency of the harvester and $V_{C}$ refers to the capacitor voltage Provided that the number of bands depends on the capacitor voltage, switching to additional bands requires additional voltage levels. Unfortunately, charging a capacitor to a higher voltage has an associated quadratic loss of energy. Accordingly, the energy required to switch from one band to another is given by:

$$
E_{s w}=\frac{1}{2} C\left(\Delta V_{C}\right)^{2}
$$

where $\Delta V_{C}$ refers to the difference between voltage levels.

4) Energy Harvesting Front-End: This unit is in charge of adapting the power which is generated by the energy harvester to generate a DC current which is delivered to the energy buffer and the remaining sub-system units of a nano-micro interface or a nanosensor. As a result of this power processing 


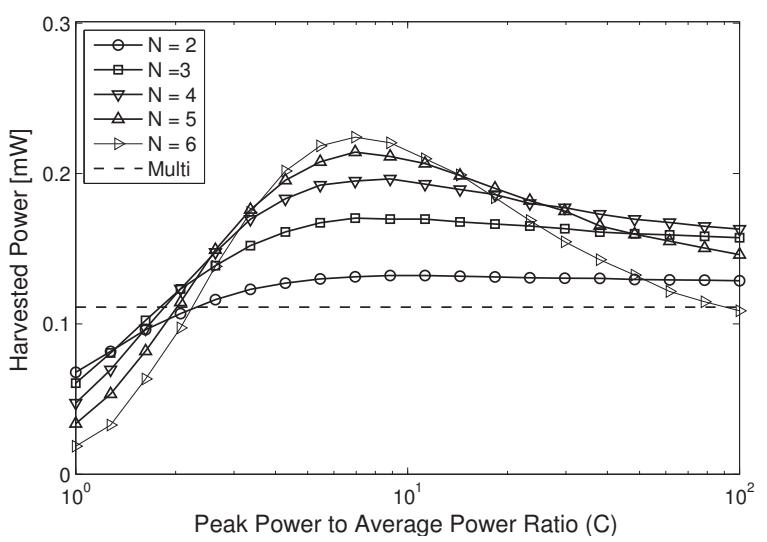

Fig. 12. Harvested power as a function of the peak power to average power ratio in self-tunable energy harvesters.

operation, the actual power which is delivered to the device is always lower than the produced by the energy harvester [4]. This is generally referred as the efficiency of the energy harvester.

\section{B. Performance Evaluation}

We evaluate the performance of a self-tunable energy harvester in terms of the average power which is able to generate. For this, we consider the energy balance at the energy harvester by calculating the generated power and the power losses derived from sensing the spectrum and retuning the harvester.

To derive the generated power, we have assumed that a self-tunable energy harvester occupies the same area as the optimized case in multi-source energy harvesting platforms and is able to generate the same power. Alternatively, we have assumed that the power that the energy harvester consumes to sense the spectrum, to process this information and to tune the oscillating frequency of the energy harvester, referred as $P_{\text {loss }}$, quadratically depends on the voltage range applied, $V_{C}$, to an equivalent capacity of $C_{e q}=1 \mu \mathrm{F}$, which is a reasonable value as reported in [10]. The voltage applied at the capacitor linearly depends to the number of frequency bands, as shown in (13).

We show in Fig. 12 the harvested power as a function of the peak power to average power ratio, $C$, for different number of available bands. In addition, we compare the results to the multi-source energy platform which has been optimized in the previous section for $C=10$ with 4 energy harvesters. In order to calculate these results, we have considered that the voltage difference to tune between consecutive bands is $0.5 \mathrm{~V}$. As the figure shows, when the peak power to average power ratio increases, the power of the energy sources is more compacted in time. Then, the likelihood that two energy sources are generating power at the same time is reduced. This permits the energy harvester to maximize the harvestable energy, thereby showing a better performance than multi-source energy harvesters. However, as this factor becomes large, the energy devoted to perform spectrum sensing and tuning the oscillating frequency gains significance, thus negatively impacting upon the performance of the energy harvester. In

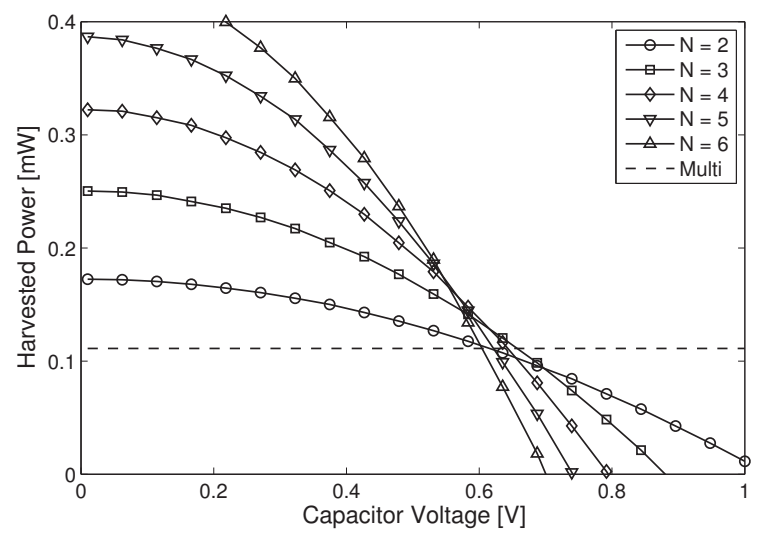

Fig. 13. Harvested power as a function of the capacitor voltage in self-tunable energy harvesters.

addition, it is observed that the number of frequency bands plays an important role in the performance of the energy harvester. In fact, considering more energy bands improve the likelihood of a given band being active, but significantly increases the power losses.

We then show in Fig. 13 the harvested power as a function of the applied voltage at the equivalent capacitor. In addition, we compare the results to the multi-source energy platform which has been optimized in the previous section for $C=10$ with 4 energy harvesters. To calculate these values, a peak power to average power ratio of $C=10$ has been assumed. As it is shown, the applied voltage has a very strong impact upon the performance of the energy harvester. In fact, as this voltage approaches zero, increasing the number of bands can provide a very large improvement compare to multi-source energy harvesting platforms. As an example, using a selftunable energy harvester to harvest from 4 bands generates almost 3 times the energy that an optimized multi-source energy harvester with the same number of bands. However, as the required capacitor voltage increases, the performance of the energy harvester is being affected, therefore showing equal performance at a capacitor voltage of approximately $V_{C}=0.65 \mathrm{~V}$. This shows the need of sophisticated sensing schemes to minimize the power consumption.

Finally, we optimize the number of bands of a self-tunable energy harvester as a function of the peak power to average power ratio and capacitor voltage in Fig. 14. In addition, this performance is compared to the performance of multi-source energy harvesting platforms. As the figure shows, regardless of the associated power losses of the energy harvesting unit, multi-source energy harvesting platforms outperforms selftunable harvesters, in terms of outage probabilities, for moderately low values of $C$. Then, as this parameter increases, the effect of the capacitor voltage becomes significant. In particular, it is observed that low number of bands show more robust performance in terms of both studied parameters, whereas considering a large number of bands require low capacitor voltages and large peak power to average power ratios. 


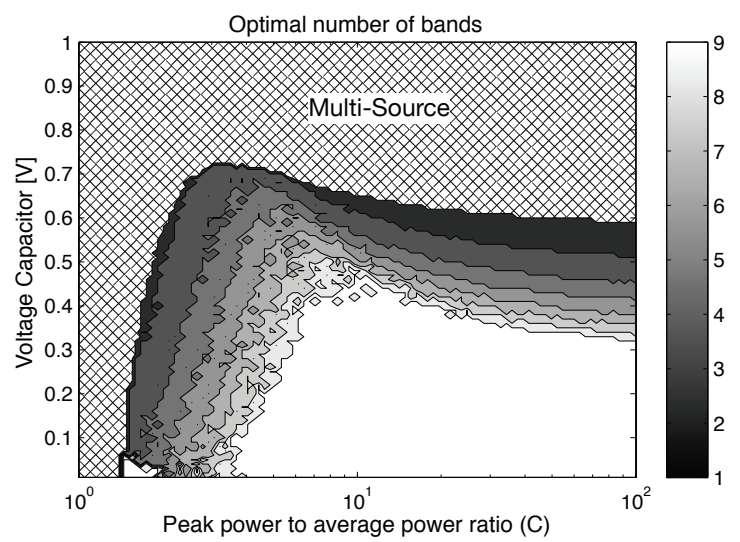

Fig. 14. Design space of self-tunable energy harvesters. Optimal number of bands as a function of the capacitor voltage and peak power to average power ratio.

\section{CONCLUSION}

Multi-source energy harvesting is gaining popularity as an alternative to power nanonetworks. The benefits that this alternative provides when the ambient energy is largely timevariant is two-fold: on the one hand, it provides robustness to the sensors and nano-micro interfaces, while on the other hand, the sparsity of the overall contribution is reduced, and thus its operation lifetime is improved. In this context, circuit area optimization which considers both energy harvester and energy buffer and takes advantage of the improvement in performance of multiple-source energy harvesters has been addressed. As it has been shown, this joint effort can help reducing the overall area, thus enabling circuit area optimization to pursue a future miniaturization of the communicating devices towards the nanoscale. In addition, the performance of self-tunable energy harvesters has been compared to an optimized multisource energy harvester. Self-tunable harvesters have shown better performance especially when the presented environmental energy is very sparse. However, the operation of these devices require sensing and computing tasks to actively select the optimal energy band.

\section{ACKNOWLEDGEMENTS}

This work has been partially funded by AGAUR FI-DGR grant.

\section{REFERENCES}

[1] R. Cid-Fuentes, A. Cabellos-Aparicio, and E. Alarcon, "Circuit area optimization in energy temporal sparse scenarios for multiple harvester powered systems," in Circuits and Systems (ISCAS), 2014 IEEE International Symposium on, June 2014, pp. 2486-2489.
[2] Y. Hu, Y. Zhang, C. Xu, L. Lin, R. L. Snyder, and Z. L. Wang, "Selfpowered system with wireless data transmission," Nano Letters, vol. 11 no. 6, pp. 2572-2577, 2011.

[3] J. Jornet and I. Akyildiz, "Joint energy harvesting and communication analysis for perpetual wireless nanosensor networks in the terahertz band," IEEE Transactions on Nanotechnology, vol. 11, no. 3, pp. 570 -580 , May 2012.

[4] R. Gomez Cid-Fuentes, A. Cabellos-Aparicio, and E. Alarcon, "Energy buffer dimensioning through energy-erlangs in spatio-temporalcorrelated energy-harvesting-enabled wireless sensor networks," IEEE Journal on Emerging and Selected Topics in Circuits and Systems, vol. 4, no. 3, pp. 301-312, Sept 2014.

[5] S. Sudevalayam and P. Kulkarni, "Energy harvesting sensor nodes: Survey and implications," IEEE Communications Surveys Tutorials, vol. 13, no. 3, pp. $443-461,2011$.

[6] D. Pech, M. Brunet, H. Durou, P. Huang, V. Mochalin, Y. Gogotsi, P.-L. Taberna, and P. Simon, "Ultrahigh-power micrometre-sized supercapacitors based on onion-like carbon," Nature Nano, vol. 5, no. 9, pp. 651 - 654, September 2010

[7] S. Bandyopadhyay and A. Chandrakasan, "Platform architecture for solar, thermal, and vibration energy combining with mppt and single inductor," IEEE Journal of Solid-State Circuits, vol. 47, no. 9, pp. 2199 -2215, September 2012.

[8] C. Park and P. Chou, "Ambimax: Autonomous energy harvesting platform for multi-supply wireless sensor nodes," in Proc. of the 3rd Annual IEEE Communications Society on Sensor and Ad Hoc Communications and Networks (SECON), vol. 1, 2006, pp. 168-177.

[9] A. S. Weddell, M. Magno, G. V. Merrett, D. Brunelli, B. M. Al-Hashimi, and L. Benini, "A survey of multi-source energy harvesting systems," in Proc. of the Design, Automation Test in Europe Conference Exhibition (DATE), 2013, pp. 905-908.

[10] C. Eichhorn, R. Tchagsim, N. Wilhelm, and P. Woias, "A smart and self-sufficient frequency tunable vibration energy harvester," Journal of Micromechanics and Microengineering, vol. 21, no. 10, p. 104003, 2011.

[11] S. Jo, M. Kim, and Y. Kim, "Passive-self-tunable vibrational energy harvester," in International Solid-State Sensors, Actuators and Microsystems Conference (TRANSDUCERS), June 2011, pp. 691-694.

[12] I. Akyildiz and J. Jornet, "The internet of nano-things," IEEE Wireless Communications, vol. 17, no. 6, pp. 58-63, December 2010.

[13] M. Win, P. Pinto, and L. Shepp, "A mathematical theory of network interference and its applications," Proceedings of the IEEE, vol. 97, no. 2, pp. $205-230$, feb. 2009.

[14] S. Chalasani and J. Conrad, "A survey of energy harvesting sources for embedded systems," in Proc. of the IEEE Southeastcon, 2008, pp. $442-447$.

[15] A. Hajati, S. Bathurst, H. Lee, and S. Kim, "Design and fabrication of a nonlinear resonator for ultra wide-bandwidth energy harvesting applications," in Proc. of the IEEE International Conference on Micro Electro Mechanical Systems (MEMS), January 2011, pp. 1301 -1304.

[16] R. G. Cid-Fuentes, A. Cabellos, and E. Alarcon, "Energy harvesting enabled wireless sensor networks: Energy model and battery dimensioning," in Proc. of the 7th International Conference on Body Area Networks (BODYNETS), September 2012.

[17] R. Rajesh, V. Sharma, and P. Viswanath, "Information capacity of energy harvesting sensor nodes," in Proc. of the IEEE International Symposium on Information Theory, 31 2011-aug. 5 2011, pp. 2363 -2367.

[18] O. Ozel, K. Tutuncuoglu, J. Yang, S. Ulukus, and A. Yener, "Transmission with energy harvesting nodes in fading wireless channels: Optimal policies," Journal of Selected Areas in Communications, vol. 29, pp. 1732-1743, September 2011.

[19] M. Gorlatova, A. Wallwater, and G. Zussman, "Networking low-power energy harvesting devices: Measurements and algorithms," in Proc. of the IEEE INFOCOM, april 2011, pp. $1602-1610$.

[20] D. Cabric, A. Tkachenko, and R. Brodersen, "Spectrum sensing measurements of pilot, energy, and collaborative detection," in IEEE Military Communications Conference,, Oct 2006, pp. 1-7. 